\title{
Experience with Orbital Tumors from a Tertiary \\ Cancer Centre of North East India: A Pathology Perspective
}

\author{
Zachariah Chowdhury Jagannath Dev Sharma² Lopa Mudra Kakoti ${ }^{2}$ Anupam Sarma² \\ Shiraj Ahmed² Munlima Hazarika ${ }^{3}$
}

\footnotetext{
${ }^{1}$ Department of Pathology, Homi Bhabha Cancer Hospital/MPMMCC (Tata Memorial Hospital), Varanasi, Uttar Pradesh, India

2Department of Pathology, Dr. B. Borooah Cancer Institute, Guwahati, Assam, India

${ }^{3}$ Department of Medical Oncology, Dr. B. Borooah Cancer Institute, Guwahati, Assam, India
}

J Lab Physicians:2020;12:171-177

\begin{abstract}
Address for correspondence Zachariah Chowdhury, MBBS, MD, Department of Pathology, Homi Bhabha Cancer Hospital/ MPMMCC (Tata Memorial Hospital), Varanasi, Uttar Pradesh, India (e-mail: chowdhury.zachariah@gmail.com).
\end{abstract}

\begin{abstract}
Keywords

- histopathology

- immunohistochemistry

- lymphoma

- orbit

- rhabdomyosarcoma

Background The orbit is an anatomically complex structure comprising the globe, extraocular muscles, fat, vascular, nervous, glandular, and connective tissues. A wide variety of neoplasms can arise from different orbital structures, which can create a diagnostic challenge to the pathologists. No formal study has been conducted in this regard in North East India.

Aim and Objectives This article aims to document the pattern and prevalence of orbital tumors in our institute and assess the utility of histopathological examination (HPE) and immunohistochemistry (IHC) in the precise diagnosis of these neoplasms.

Materials and Methods A retrospective analysis of orbital tumors was performed over a period of 5 years from 2013 to 2018 in the department of pathology at a tertiary cancer center of North East India following all the guidelines of the institutional ethics committee. Results A total of 35 cases of orbital neoplasms, evaluated by HPE and IHC, were found, all of them being malignant tumors. The age range was 4 months to 85 years. Male to female ratio was 1.5:1. The most common tumor found was lymphoma, accounting for 10 cases (28.6\%), all of which were non-Hodgkin lymphoma (NHL). All these cases except one occurred in adults, thus making it the most common tumor in adults in this study. Diffuse large B cell lymphoma, not otherwise specified, was the most common NHL, followed by follicular lymphoma, mature T cell NHL, extranodal marginal zone lymphoma, and B cell lymphoblastic lymphoma. Rhabdomyosarcoma and poorly differentiated/ undifferentiated carcinoma jointly were the second most common tumors, totaling seven cases (21.21\%) each. This was followed by melanoma (three cases), myeloid sarcoma (three cases), Ewing sarcoma/peripheral neuroectodermal tumor (PNET) (three cases), neuroblastoma (one case), and angiosarcoma (one case). Among these, rhabdomyosarcoma, granulocytic sarcoma, Ewing sarcoma/PNET, and neuroblastoma exclusively troubled the children. IHC markers including the lymphoma panel, and soft tissue ones were crucial in the precise diagnosis of the neoplasms encountered.
\end{abstract}

DOI https://doi.org/ 10.1055/s-0040-1721148 ISSN 0974-2727 (c) 2020. The Indian Association of Laboratory Physicians.

This is an open access article published by Thieme under the terms of the Creative Commons Attribution-NonDerivative-NonCommercial-License, permitting copying and reproduction so long as the original work is given appropriate credit. Contents may not be used for commercial purposes, or adapted, remixed, transformed or built upon. (https://creativecommons.org/licenses/by-nc-nd/4.0/)

Thieme Medical and Scientific Publishers Pvt. Ltd., A-12, 2nd Floor, Sector 2, Noida-201301 UP, India 
Conclusion A variety of malignant orbital tumors may be seen in clinical practice. Management of these tumors requires a multidisciplinary approach. HPE in conjunction with IHC evaluation is of utmost importance in the veracious recognition of orbital tumors for their proper management.

\section{Introduction}

Orbital malignancies are an important group of disorders presenting in all age groups. Malignant tumors of the orbit may originate either from the primary orbital tissues including the eyeball, or may invade from surrounding structures like eyelids, paranasal sinuses, nasopharynx, or cranial cavity (secondary tumors). They may also be a presentation of systemic lymphoproliferative disorders or metastasis from malignant tumors elsewhere in the body. ${ }^{1}$ The histopathological characteristics of these tumors are critical to their biologic behavior, line of management, outcome, and prognosis. Thus, accurate diagnosis of these tumors based on histopathological examination (HPE) and immunohistochemistry (IHC) is the sine qua non for definitive management.

\section{Aims and Objectives}

The study was conducted to study the pattern and prevalence of orbital tumors in our institute, and to assess the utility of HPE and IHC in correctly diagnosing these neoplasms.

\section{Material and Methods}

A retrospective analysis of orbital tumors was performed over a period of 5 years from 2013 to 2018 in the department of pathology at a tertiary cancer care center following all the guidelines of the institutional ethics committee. Only those cases with conclusive and unequivocal diagnosis, evaluated by both HPE and IHC were included in the study. Details of age, gender, and other relevant clinical information were collected from the medical records. The hematoxylin and eosin (H\&E) stained slides sectioned from formalin-fixed paraffin-embedded tissues and the IHC stained slides were retrieved from the archives in the department of pathology and evaluated.

\section{Results}

A total of 35 cases of orbital tumors, evaluated by HPE and IHC were discovered, all of them being unilateral and malignant in nature. None of the tumors except one were metastatic in origin. Common signs and symptoms encountered were orbital swelling, proptosis, vision impairment, pain, restricted motility, leukocoria (white pupil), and ptosis. The age range was 4 months to 85 years. Adult cases amounted to 20 , while pediatric ones accounted for 15 of the total. There were a dozen cases in the elderly ( $\geq 60$ years) out of the 20. Male to female ratio was 1.5:1 ( - Table $\mathbf{1}$ ).

The most common tumor found was lymphoma, accounting for 10 cases (28.6\%), all of which were non-Hodgkin lymphoma (NHL) (-Table 2). All these cases except one occurred in adults (nine cases), thus making it the most common tumor not only in adults, but also in the elderly ( $\geq 60$ years). The structure of origin of these neoplasms was variable, a few arising from the lacrimal sac and/or lacrimal gland while the source of the others could not be precisely ascertained (nonlacrimal origin). B cell NHLs constituted the majority of the lymphomas, being extranodal in nature. Diffuse large B cell lymphoma (DLBCL), not otherwise specified (NOS), was the most common lymphoma (four cases), followed by follicular lymphoma (two cases), mature T cell NHL (two cases), extranodal marginal zone lymphoma (EMZL), and B cell lymphoblastic lymphoma (one case each). All the DLBCL cases occurred in adults, age range being 30 to 71 years (-Fig. 1). Among these, all except one case belonged to the activated

Table 1 Clinicopathologic profile of the orbital tumors in the study

\begin{tabular}{|c|c|c|c|c|c|c|c|c|c|}
\hline \multirow{2}{*}{$\begin{array}{l}\text { SI } \\
\text { no. }\end{array}$} & \multirow[t]{2}{*}{ Diagnosis } & \multirow{2}{*}{$\begin{array}{l}\text { No of } \\
\text { cases }\end{array}$} & \multirow[t]{2}{*}{ Age range } & \multirow[t]{2}{*}{ Pediatric } & \multicolumn{2}{|c|}{ Adult } & \multirow[t]{2}{*}{ Male } & \multirow[t]{2}{*}{ Female } & \multirow{2}{*}{$\begin{array}{l}\text { Incidence } \\
(\%)\end{array}$} \\
\hline & & & & & $<60 y$ & $\geq 60 y$ & & & \\
\hline 1. & $\mathrm{NHL}$ & 10 & $6-71 y$ & 01 & 03 & 06 & 07 & 03 & 28.57 \\
\hline 2. & RMS & 07 & $1-16 y$ & 07 & 0 & 0 & 03 & 04 & 20.0 \\
\hline 3. & PDC/UC & 07 & $24-85 y$ & 00 & 03 & 04 & 05 & 02 & 20.0 \\
\hline 4. & Melanoma & 03 & $42-75 y$ & 00 & 01 & 02 & 03 & 00 & 8.57 \\
\hline 5. & Myeloid sarcoma & 03 & $2-10 y$ & 03 & 0 & 0 & 02 & 01 & 8.57 \\
\hline 6. & Ewing/PNET & 03 & $3-15 y$ & 03 & 0 & 0 & 01 & 02 & 8.57 \\
\hline 7. & Neuroblastoma & 01 & $4 \mathrm{mo}$ & 01 & 0 & 0 & 0 & 01 & 2.86 \\
\hline 8. & Angiosarcoma & 01 & $32 y$ & 0 & 01 & 0 & 0 & 01 & 2.86 \\
\hline 9. & Total & 35 & $4 \mathrm{mo}-85 \mathrm{y}$ & 15 & 08 & 12 & 21 & 14 & 100 \\
\hline
\end{tabular}

Abbreviations: NHL, non-Hodgkin Lymphoma; PDC/UC, poorly differentiated carcinoma/undifferentiated carcinoma; PNET, peripheral neuroectodermal tumor; RMS, rhabdomyosarcoma. 
Table 2 Histologic subtypes of the lymphomas in the study

\begin{tabular}{|l|l|l|l|l|l|l|l|l|l|}
\hline $\begin{array}{l}\text { SI } \\
\text { no. }\end{array}$ & $\begin{array}{l}\text { NHL } \\
\text { subtype }\end{array}$ & $\begin{array}{l}\text { No of } \\
\text { cases }\end{array}$ & $\begin{array}{l}\text { Age } \\
\text { range }(y)\end{array}$ & Pediatric & Adult & Male & Female & $\begin{array}{l}\text { Clinical } \\
\text { stage }\end{array}$ & $\begin{array}{l}\text { Percentage } \\
\text { (\%) }\end{array}$ \\
\hline 1. & DLBCL & 04 & $30-71$ & 0 & 04 & 03 & 01 & II, III & 40 \\
\hline 2. & $\begin{array}{l}\text { Follicular } \\
\text { NHL }\end{array}$ & 02 & 60,65 & 0 & 02 & 01 & 01 & III & 20 \\
\hline 3. & PTCL NOS & 02 & 55,60 & 0 & 02 & 02 & 0 & II & 20 \\
\hline 4. & $\begin{array}{l}\text { Marginal } \\
\text { Zone NHL }\end{array}$ & 01 & 63 & 0 & 01 & 0 & 01 & I & 10 \\
\hline 5. & BLL & 01 & 6 & 01 & 0 & 01 & 0 & II & 10 \\
\hline 6. & Total & 10 & $6-71$ & 01 & 09 & 07 & 03 & I-III & 100 \\
\hline
\end{tabular}

Abbreviations: BLL, B cell lymphoblastic lymphoma; DLBCL, diffuse large B cell lymphoma; NHL, non-Hodgkin Lymphoma; PTCL NOS, peripheral T cell lymphoma not otherwise specified.
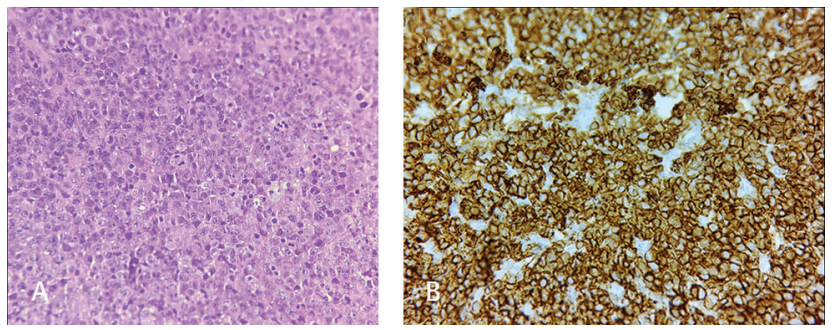

Fig. 1 Photomicrograph of diffuse large B cell lymphoma (DLBCL) showing proliferation of atypical medium sized lymphoid cells in diffuse sheets (A) (hematoxylin and eosin [H\&E], 40×), which are positive for CD20 on immunohistochemistry (IHC) (B) (40×).

B cell (ABC) subtype on the basis of Hans algorithm, thus demonstrating positivity for $\mathrm{ABC}$ marker such as Mum1, while being negative for germinal center markers such as CD10 and bcl6. Three of these had stage II disease while one was in stage III (Ann Arbor staging). Follicular lymphoma was identified to involve the elderly ( $\geq 60$ years), and both the cases belonged to grade 2 histomorphologically, and clinical stage III. The lacrimal tissue was the origin of the EMZL in a 63-year-old lady, whose clinical stage was stage I. The only lymphoma detected in the pediatric population was a case of B cell lymphoblastic lymphoma, which affected a 6 -year-old boy and presented in clinical stage II. The differentiating feature from Burkitt lymphoma was the unequivocal TdT positivity on IHC and Ki67 index of approximately $90 \%$. $\mathrm{T}$ cell orbital lymphomas discovered in the study were of the peripheral $\mathrm{T}$ cell type (NOS), exclusively occurring in adults and presenting in clinical stage II. The characteristic immunophenotype of $\mathrm{CD} 3+/ \mathrm{CD} 4+/ \mathrm{CD} 8$-accompanied by downregulation of other $\mathrm{T}$ cell markers such CD7 and CD5 suggested the diagnosis. CD30 and Alk1 negativity in the atypical lymphoid cells excluded the possibility of anaplastic large cell lymphoma in these two cases. It was interesting to note that none of the lymphoma cases revealed bone marrow involvement. Chemotherapy was the main modality of therapy as most of the tumors presented in stage II or higher, and the regimen employed was cyclophosphamide, vincristine, doxorubicin, and prednisolone, with or without immunotherapy such as rituximab. The only localized lymphoma in the study, namely EMZL, was managed by radiation therapy.
Rhabdomyosarcoma (RMS) and poorly differentiated carcinoma (PDC)/undifferentiated carcinoma (UC) were the second most common orbital tumors comprising 7 cases each. The muscular tissues of the orbit were the source of the RMS, while PDC/UC arose primarily from the lacrimal gland. All the patients of RMS were children, while PDC/ UC affected only adults over a wide age range, the youngest being 24 years and the oldest 85 years. Histology of PDC/UC displayed sheets of medium to large round to polygonal atypical cells demonstrating positivity only for pan-cytokeratin (PanCK) and epitheleal membrane antigen, while all other markers including those utilized for malignancy of unknown origin such as leukocyte common antigen (LCA), vimentin, S100, HMB45, synaptophysin, chromogranin, and CD56 were negative. The most common childhood orbital tumor was RMS. The predominant subtype was embryonal RMS (six cases), while one case belonged to alveolar histology. The tumor cells demonstrated strong positivity for desmin (cytoplasmic) and myogenin (nuclear) (-Fig. 2). No cytogenetic analysis or molecular test could be attempted. Most of these patients of RMS were treated with combination chemotherapy (vincristine + actinomycin D + cyclophosphamide/etoposide) and conventional fractionated radiation. Melanoma was the other predominant orbital malignancy in adults, genesis being the choroid. Melanin pigment was perceptible in the H\&E sections. S100 and HMB45 IHC highlighted the atypical cells and corroborated the diagnosis. These patients were treated with plaque brachytherapy.

Myeloid sarcoma (MS) involved the orbit in three pediatric patients (age 2-10 years). This was the initial presentation in these patients before the documentation of any systemic disease. HPE showed a picture of small round cell tumor; the finding of eosinophilic myelocytes on histology provided a hint of granulocytic sarcoma. IHC evaluation was crucial in pinpointing the diagnosis. The tumor showed positivity for LCA, CD34, CD68, and most importantly myeloperoxidase (MPO) ( - Fig. 3). The patient was treated with cytarabine and daunorubicin $(7+3$ regimen) induction chemotherapy, and later consolidation chemotherapy with high-dose cytarabine for four cycles. The patient remained in complete remission 24 months since diagnosis. Another three pediatric patients were diagnosed with Ewing sarcoma (ES)/peripheral 

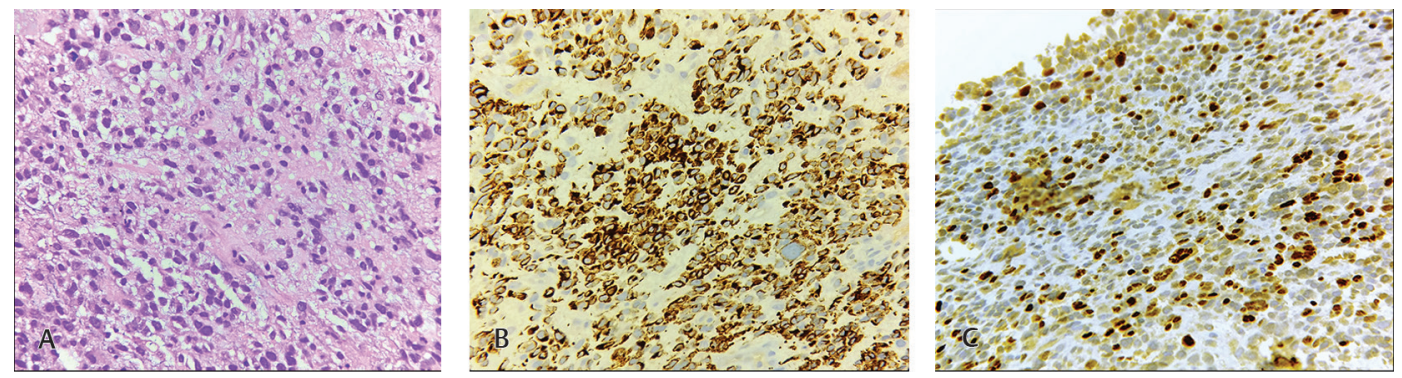

Fig. 2 Photomicrograph of rhabdomyosarcoma (RMS) exhibiting sheets of small round cells, some of which have a plasmacytoid appearance (A) (hematoxylin and eosin [H\&E], 40×). Immunohistochemistry (IHC) displays positivity of the tumor cells for desmin (B, 40X×), and myogenin (C, 40×).
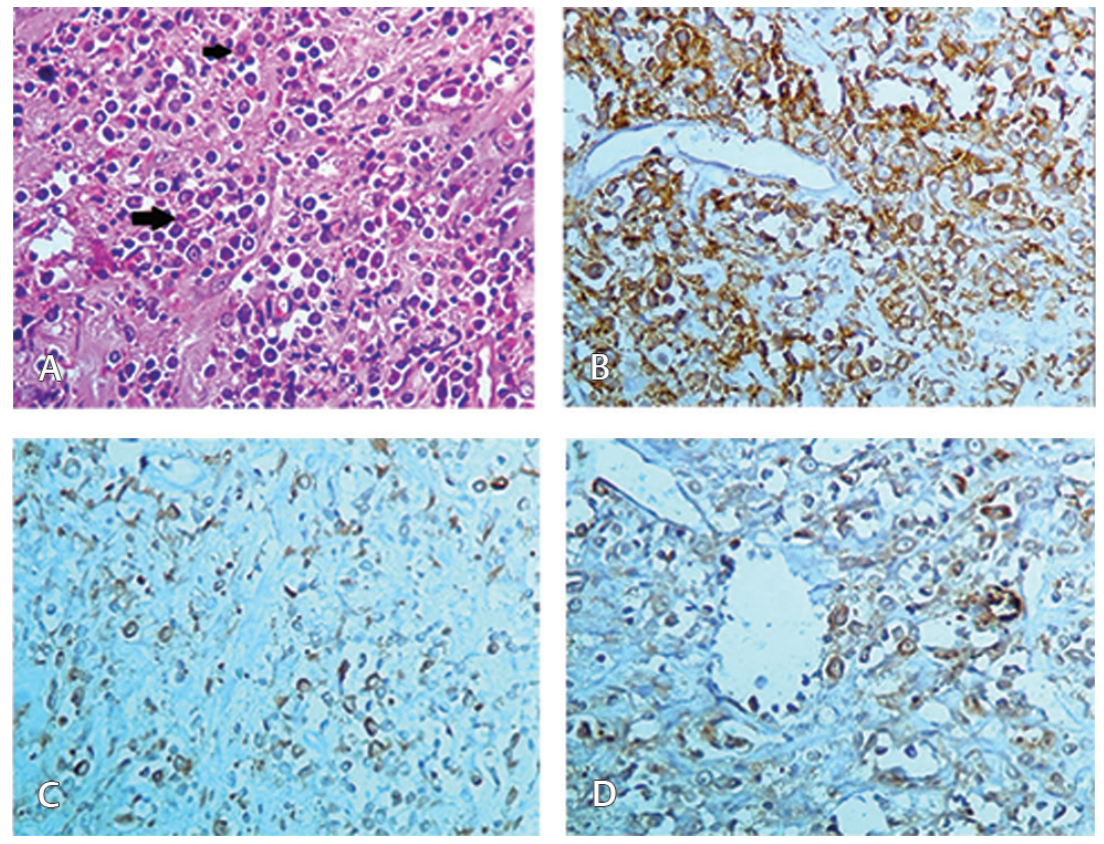

Fig. 3 Photomicrograph of myeloid sarcoma consisting of blasts having round to oval nuclei, fine chromatin, and small distinct nucleoli and scant cytoplasm, accompanied by a few eosinophils and its precursors (arrows) (A) (hematoxylin and eosin [H\&E], 40x). Immunohistochemistry (IHC) pinpoints to the diagnosis by the positivity of the blasts for leukocyte common antigen (LCA) (B), myeloperoxidase (MPO) (C), and CD68 (D) (40×).
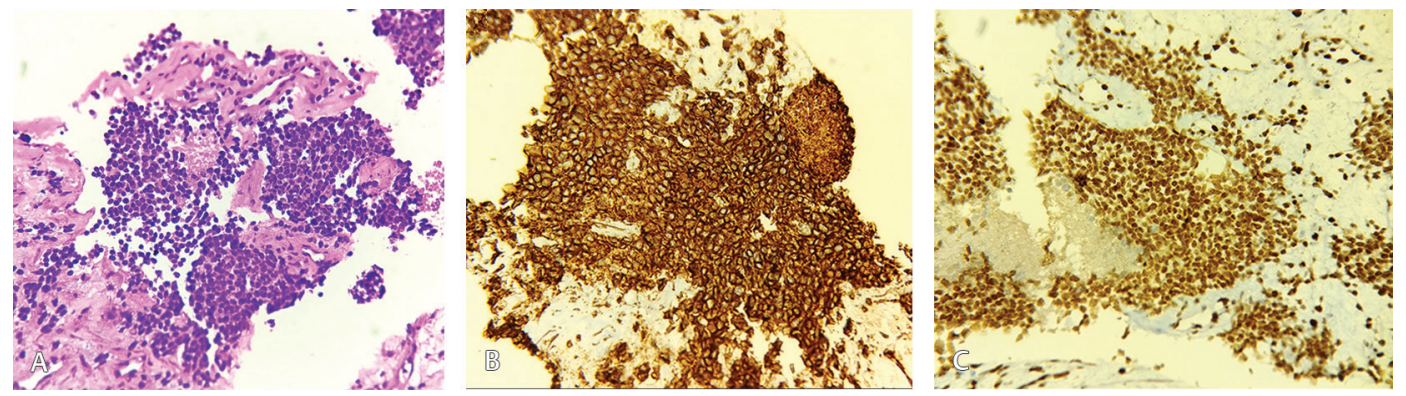

Fig. 4 Photomicrograph of Ewing sarcoma/peripheral neuroectodermal tumor (PNET) composed of sheets of small round cells (A) (hematoxylin and eosin [H\&E], 40×), manifesting complete membranous positivity for CD99 (B, 40×), and nuclear positivity for FLI1(C) (40×).

neuroectodermal tumor (PNET); the tumors consisting of sheets of small round atypical cells. Complete membranous positivity for CD99, diffuse strong nuclear FLI1 positivity, and negativity for other markers such as LCA, desmin, synaptophysin, and PanCK clinched the diagnosis ( - Fig. 4). After the complete metastatic workup, the patients were treated on nonmetastatic Ewing family tumor chemotherapy protocol with intensity-modulated radiotherapy. All the three patients after completion of treatment were alive without any evidence of recurrence at 48, 33, and 26 months until last follow-up. 

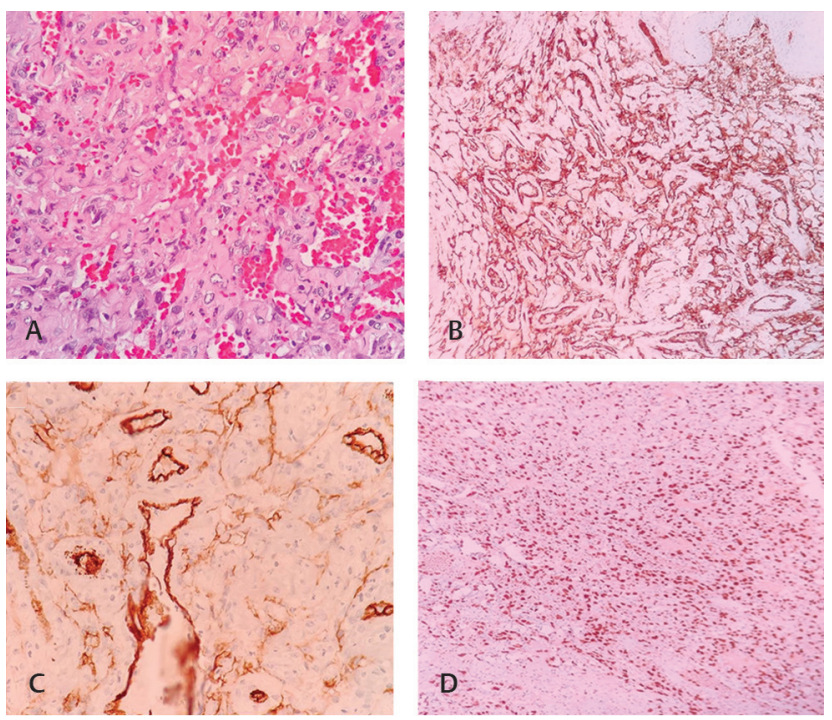

Fig. 5 Photomicrograph of angiosarcoma of the eyelid revealing anastomosing vascular spaces lined by atypical endothelial cells (A) (hematoxylin and eosin [H\&E], 40×), highlighted by immunoreactivity for CD31 (B), CD34 (C), and FLI1 (D) (40×).

A sole case of neuroblastoma involving the orbit was encountered. Histology was of poorly differentiated type; neuropil, Homer-Wright pseudorosettes surrounding eosinophilic neuropil, and occasional cells displaying features of differentiating neuroblasts $(<5 \%)$ were discernible. Variable positivity for synaptophysin and chromogranin was noted, while being negative for other markers like CD99, LCA, PanCK, FLI1, and desmin. Systemic workup revealed primary in the abdomen, involving the orbit as a secondary. The patient was treated with combination chemotherapy comprising vincristine, Adriamycin, and cyclophosphamide. After completion of three cycles, there was significant reduction of tumor volume, and the child was systemically well. The last case in the list was a rare case of angiosarcoma of the eyelid diagnosed in a 32-year-old lady. CD34, CD31, and FLI1 positivity underlined the vascular origin of the atypical cells ( - Fig. 5). Absence of any other diagnostically similar lesion elsewhere in the body confirmed the primary nature of the angiosarcoma. Subsequent wide surgical resection was performed, with substantial reconstruction. The patient responded well to the surgery, and was free of tumor after 15 months of follow-up. Genderwise, the most common malignancy in males was NHL with seven cases, while RMS constituted the majority in females with four cases. The notable exceptions in the list of diagnosis were retinoblastoma and inflammatory myofibroblastic tumor, no cases of which were encountered.

\section{Discussion}

Orbital tumors can occur at various age groups, and because of their myriad presentation, their diagnosis pose a sound challenge. The reported incidence of orbital tumors varies widely from series to series. A review of the literature revealed marked differences between biopsy-proven studies, and clinically based studies. ${ }^{2}$ Nonetheless, overall incidence of orbital tumors is 3.5 to $4 \%{ }^{3}$ The major bias inherent in our study was the nature of the institute where the research was taken up. Being a tertiary cancer care center dedicated to all disciplines without the services of any ophthalmologist, two points of prejudice were deciphered. First, no rendezvous with benign neoplasms and inflammatory lesions, and second, tumors such as retinoblastoma and/or inflammatory myofibroblastic tumor were noticeable omissions as the specialized eye centers in the locality catered to these. Nevertheless, the present study was a sincere attempt to pathologically characterize the orbital neoplasms, the first of its kind from the North East part of India.

A study conducted in Dublin and few in India observed benign lesions to be greater than the malignant tumors. ${ }^{4,5}$ However, a few other studies found malignant tumors to be preponderant. ${ }^{6}$ The study from Dublin reported secondary tumors to be the most common source whereas the present study reports primary orbital tissue to be the most common source of orbital tumors as was also observed in other studies. ${ }^{1}$ The main clinical features at presentation include mass (palpable or visualized), proptosis, pain, defective vision, restricted mobility, leukocoria, and ptosis., 3,8

Orbital lymphoma is the most common malignant tumor found in the orbit ranging from 4 to 13\%. The vast majority of orbital lymphomas are of B cell origin (97\%), of which EMZL (59\%) is the most common subtype, followed by DLBCL (23\%), follicular lymphoma (9\%), and mantle cell lymphoma (5\%). Primary orbital lymphoma remains a rare entity, comprising only approximately $1 \%$ of NHLs. Primary orbital lymphoma is when the patient presents with no other concurrent systemic lymphoma or prior history of lymphoma..$^{9}$ The case of EMZL being a stage I disease justified as a primary orbital lymphoma in our series. The other lymphomas though extranodal in nature had concurrent nodal disease as well. Orbital lymphoma is primarily a disease of the elderly. Gender distribution varies according to lymphoma subtype. EMZL (53\%) and follicular lymphoma (75\%) show a female predominance, whereas DLBCL shows an even gender distribution. ${ }^{10}$ It typically presents in patients older than 50 years which is almost similar to mean age group of orbital lymphoma in general which is 50 to 70 years old. In our study, the most common NHL was DLBCL NOS, unlike the finding in the other studies, wherein MZL was the most common. ${ }^{9,11,12}$ The other parameters mentioned above such as age and gender distribution conformed to our series. DLBCL, NOS can be subdivided into germinal center $B$ cell subtype and ABC subtype. This determination of cell of origin has begun to penetrate clinical practice and is therefore required. If gene expression technologies are not available as was the situation in our series, then IHC analysis is considered an acceptable alternative. ${ }^{13}$ Most extranodal DLBCLs seem to be of the ABC subtype, as was also evident in our series. Primary orbital/ocular adnexal T cell extranodal lymphoma is rare and most cases represent secondary orbital involvement by distant spread of systemic disease. ${ }^{14}$ At the time of diagnosis, peripheral lymphadenopathy is common and visceral involvement is unusual. The diagnosis relies on recognition of a characteristic histology 
with a diffuse growth pattern, lymphoid cells with intermediate nuclear size, fine chromatin, indistinct nucleoli, and frequent mitotic figures, and characteristic IHC pattern, namely aberrant loss of $\mathrm{T}$ cell antigens. ${ }^{14,15}$

The most common biopsied malignant tumor of the orbit in children is RMS, and comprises $4 \%$ of all pediatric malignancies, with $10 \%$ of all cases occurring in the orbit. ${ }^{16}$ Ninety percent of primary orbital RMS occurs before the age of 16 years with a mean age of 5 to 7 years. Males are slightly more affected than females, with male to female ratio of 5:3. ${ }^{17}$ In our series, the age range was quite similar, while females slightly outnumbered males. Majority of orbital RMS is of the embryonal type (50-70\%). Alveolar RMS often harbors a typical translocation, but embryonal RMS lacks any specific rearrangement. So, histopathology is not always sufficient for an unequivocal diagnosis, necessitating ancillary studies. ${ }^{2}$ IHC studies constitute the main approach to diagnosis. The markers typically found in RMS include antibodies against desmin (90\%), myogenin (90\%), myoD1 (71-91\%), and myoglobin. Myogenin (90\%) is expressed more in the alveolar than in the embryonal type, while myoD1 is preferentially expressed in the embryonal type. Desmin is usually positive but less specific since it can be positive in other tumors with skeletal muscle differentiation. ${ }^{16}$

MS is a rare extramedullary tumorous aggregation of malignant myeloid precursor cells and may occur as a manifestation of a well-established systemic myelogenous leukemia or it may precede systemic manifestations of peripheral blood and bone marrow, as was the condition in our study. MS more commonly occurs in the pediatric population and has most commonly been reported in skin, bone, and lymph nodes, but it is well accepted that it may occur at almost any location in the body including the orbit. ${ }^{18}$ Establishing the diagnosis of MS presenting de novo continues to challenge clinicians. Even in cases where MS occurs in the setting of previously diagnosed acute myeloid leukemia (AML), imaging cannot differentiate this from other possible complications associated with AML. For this reason, a tissue sample is needed for accurate diagnosis. Even on histopathology, the differential diagnosis is wide, ranging from various types of NHLs, small round cell tumors (in particular in children), UCs, soft tissue sarcomas to rare entities like histiocytic/dendritic cell neoplasms, or blastic plasmacytoid dendritic cell neoplasm. ${ }^{19}$ Appropriate IHC staining in these cases is particularly essential to establish the diagnosis accurately. Reports have noted several reliable markers such as CD43, CD65/KP1, MPO, CD117, and CD68.

Among the pediatric malignancies, ES/PNET and neuroblastoma are differential diagnosis of each other. Primary orbital ES/PNET is extremely rare with isolated case reports and small case series reported in literature. ${ }^{20}$ Cases with classical morphologic features can be accurately diagnosed using light microscopy with ancillary IHC. However, given the wide morphologic spectrum, genetic confirmation of nonrandom translocations leading to the fusion of the EWSR1 gene on $22 \mathrm{q} 12$ with one of several members of the ETS family of transcription factors is essential for the diagnosis of unusual morphologic variants of ES/PNET. Although initially believed to be highly specific for ES/PNET, it is apparent that virtually all other round cell tumors in the differential diagnosis, on occasion, show membranous immunoreactivity for CD99. Therefore, although CD99 is highly sensitive for recognizing ES/PNET, this marker should always be used as part of a panel of immunostains because it lacks specificity. ${ }^{21}$ FLI1 nuclear positivity has been reported in 71 to $84 \%$ of ES/PNET cases. ${ }^{22,23}$ Despite the sensitivity of this marker, FLI1 is also frequently positive in lymphoblastic and other NHLs, and rare examples of melanoma, Merkel cell carcinoma, and neuroblastoma. ${ }^{22-24}$ Neuroblastoma represents the second most common orbital tumor in children after RMS, and only $8 \%$ cases first present with an orbital lesion. ${ }^{25}$ Orbital neuroblastoma in children commonly occurs due to metastasis with primary in the abdomen. IHC plays an important role in differentiating neuroblastoma from other small round cell tumors such as retinoblastoma, RMS, and lymphoma. Tumors cells are stained for neuronal markers such as neuron-specific enolase, chromogranin, and synaptophysin which aids in the diagnosis of neuroblastoma. ${ }^{26}$ However, unlike ES/ PNET, neuroblastomas do not express CD99, and cytogenetically lack evidence of EWSR1 aberrations.

Angiosarcoma represents one of the rarest soft tissue neoplasms, comprising less than $1 \%$ of all sarcomas. Primary eyelid involvement is rare, with less than 30 cases reported in the literature. ${ }^{27}$ The rarity of ocular adnexal angiosarcoma further contributes to the clinical diagnostic challenge, with about one-quarter of the lesions initially misdiagnosed as basal cell carcinoma or pyogenic granuloma. ${ }^{28}$ The histomorphology can be variable, ranging from highly differentiated tumors resembling benign or intermediate grade vascular lesions such as hemangioma or hemangioendothelioma, to anaplastic ones difficult to distinguish from a PDC or other high grade sarcomas. IHC confirmation of the diagnosis of angiosarcoma, even those that are poorly differentiated, can usually be accomplished using a panel of vascular markers such as CD34, CD31, FLI1, factor VIII, and ERG. Among these, CD31 has been shown to be the most sensitive and most specific endothelial marker that reacts rarely and only weakly with nonvascular tumors. ${ }^{29}$

As regards the management of orbital malignancies, as observed in this study, depending on the particular type of the tumor, the localization, and the extent, the treatment options vary from chemotherapy, chemotherapy combined with radiotherapy, excision, excision followed by radiation therapy, to radical surgeries such as orbital exenteration, exenteration with radiotherapy, and exenteration with radiation therapy and chemotherapy.

There are limitations in our study, it being retrospective in nature within a single tertiary cancer center. Our study may not represent the routine usual cases that would be encountered by a general ophthalmologist. Some of the simple orbital lesions such as the cystic ones might have been managed locally while a few neoplastic entities such as retinoblastoma were treated at a specialized eye care institute. 


\section{Conclusion}

A variety of malignant orbital tumors may be seen in clinical practice. The incidence of any particular orbital neoplasm depends mainly on the type of study (clinical vs. histopathologic), medical specialty of the author, and nature of the institute conducting the study. HPE coupled with IHC is indispensable in segregating the different morphologic types of orbital tumors. This is of utmost significance for selective management of these tumors because of the differences in outcome.

\section{Funding \\ None.}

\section{Presentation at a Meeting}

None.

\section{Statement of Ethics}

The study followed all the guidelines of the institutional ethics committee and adhered to the principles of the Declaration of Helsinki.

\section{Conflict of Interest}

None.

\section{Acknowledgment}

The authors acknowledge the help of Prof. (Dr.) Amal C. Kataki, Director of the institute for guiding us through the work.

\section{References}

1 Khan AA, Sarwar S, Sadiq MAA. Ahmad I, Tariq N, Sibghat-Ul-Noor. Analysis of orbital malignancies presenting in a tertiary care hospital in Pakistan. Pak J Med Sci 2017; 33(1):70-74

2 Abdallah AM, Abdellatif MA, Elhwary AM, et al. Pediatric orbital tumours in upper Egypt: a 3-year retrospective analysis at a university hospital. J Clin Ophthalmol 2019;3(1): 108-120

3 Modi PJ, Shah NA, Bhalodia JN, Gonsai RN. Orbital tumours in children: a descriptive study at tertiary care centre. Natl J Med Res 2013;3(4):362-366

4 Shields JA, Shields CL, Scartozzi R. Survey of 1264 patients with orbital tumors and simulating lesions: the 2002 Montgomery Lecture, part 1. Ophthalmology 2004;111(5):997-1008

5 Shaikh IY, Shah FR, Gandhi MB, Shah CK, Shah NR. Ophthalmic neoplastic lesions - a retrospective study of 4 years. Gujarat Med J 2012;67(2):53-57

6 Umar AB, Ochicha O, Iliyasu Y. A pathologic review of ophthalmic tumors in Kano, Northern Nigeria. Niger J Basic Clin Sci 2012;9:23-26

7 Radha J, Sreedhar A. Orbital tumors - a clinicopathological study. Kerela J Ophthalmol 2005;3:261-265

8 Demirci H, Shields CL, Shields JA, Honavar SG, Mercado GJ, Tovilla JC. Orbital tumors in the older adult population. Ophthalmology 2002;109(2):243-248

9 Sriram PR. A rare case of aggressive, huge primary orbital lymphoma with intracranial extension and bone invasion. Asian J Neurosurg 2017;12(4):766-768
10 Olsen TG, Heegaard S. Orbital lymphoma. Surv Ophthalmol 2019;64(1):45-66

11 Eckardt AM, Lemound J, Rana M, Gellrich NC. Orbital lymphoma: diagnostic approach and treatment outcome. World J Surg Oncol 2013;11:73-78

12 Stefanovic A, Lossos IS. Extranodal marginal zone lymphoma of the ocular adnexa. Blood 2009;114(3):501-510

13 Gascoyne RD, Campo E, Jaffe ES, et al. Diffuse large B cell lymphoma, NOS. In: Swerdlow SH, ed. WHO Classification of Tumors of Haematopoietic and Lymphoid Tissues, Revised. 4th ed. Lyon: IARC; 2017 291-297

14 Janatpour KA, Choo PH, Lloyd WC II. Primary orbital peripheral T-cell lymphoma: histologic, immunophenotypic, and genotypic features. Arch Ophthalmol 2007;125(9):1289-1292

15 LeeDS, WooKI,ChangHR.T-cell lymphoma presenting as painful ophthalmoplegia. Korean J Ophthalmol 2006;20(3):192-194

16 Jurdy L, Merks JHM, Pieters BR, et al. Orbital rhabdomyosarcomas: a review. Saudi J Ophthalmol 2013;27(3):167-175

17 Malu KN, Ngbea JA, Mohammand H. Primary orbital rhabdomyosarcoma in an 11-year-old boy: a management challenge in a resource limited environment. J Med Trop 2015;17:37-41

18 Payne C, Olivero WC, Wang B, et al. Myeloid sarcoma: a rare case of an orbital mass mimicking orbital pseudotumor requiring neurosurgical intervention. Case Rep Neurol Med 2014;2014:395196

19 Pilleri SA, Orazi A, Falini B, Myeloid sarcoma. In: Swerdlow SH, et al ed. WHO Classification of Tumors of Haematopoietic and Lymphoid Tissues, Revised. 4th ed. Lyon: IARC; 2017: 167-168

20 Kaliki S, Rathi SG, Palkonda VAR. Primary orbital Ewing sarcoma family of tumors: a study of 12 cases. Eye (Lond) 2018;32(3):615-621

21 Goldblum JR, Folpe AL, Weiss SR, Malignant soft tissue tumors of uncertain type. In: Goldblum JR, Folpe AL, Weiss SR, eds. Enzinger and Weiss's Soft Tissue Tumors. 6th ed. Philadelphia: Elsevier Saunders; 2014:1028-1045

22 Llombart-Bosch A, Machado I, Navarro S, et al. Histological heterogeneity of Ewing's sarcoma/PNET: an immunohistochemical analysis of 415 genetically confirmed cases with clinical support. Virchows Arch 2009;455(5):397-411

23 Folpe AL, Hill CE, Parham DM, O'Shea PA, Weiss SW. Immunohistochemical detection of FLI-1 protein expression: a study of 132 round cell tumors with emphasis on CD99-positive mimics of Ewing's sarcoma/primitive neuroectodermal tumor. Am J Surg Pathol 2000;24(12):1657-1662

24 Rossi S, Orvieto E, Furlanetto A. Laurino L, Ninfo V, Dei Tos AP. Utility of the immunohistochemical detection of FLI-1 expression in round cell and vascular neoplasm using a monoclonal antibody. Mod Pathol 2004;17(5):547-552

25 Vallinayagam M, Rao VA, Pandian DG, Akkara JD, Ganesan N. Primary orbital neuroblastoma with intraocular extension. Indian J Ophthalmol 2015;63(8):684-686

26 Mirzai H, Baser EF, Tansug N, Nese N, Isisag A. Primary orbital neuroblastoma in a neonate. Indian J Ophthalmol 2006;54(3):206-208

27 Milman T, Shields CL, Brooks JS, et al. Primary cutaneous angiosarcoma of the eyelid: a diagnostic and therapeutic challenge. Ocul Oncol Pathol 2018;4(4):230-235

28 Lemanski N, Farber M, Carruth BP, Wladis EJ. Primary adnexal angiosarcoma masquerading as periorbital hematoma. Surv Ophthalmol 2014;59(6):655-659

29 Goldblum JR, Folpe AL, Weiss SR, Malignant vascular tumors. In: Goldblum JR, Folpe AL, Weiss SR, eds. Enzinger and Weiss's Soft Tissue Tumors. 6th ed. Philadelphia: Elsevier Saunders 2014:703-717 